\title{
NONSEARCH PARADIGM FOR LARGE-SCALE PARAMETER-IDENTIFICATION PROBLEMS IN DYNAMICAL SYSTEMS RELATED TO ONCOGENIC HYPERPLASIA
}

\author{
E. Mamontov ${ }^{1}$ and A. Koptioug ${ }^{2}$ \\ ${ }^{1}$ Department of Physics, Gothenburg University, Kemivägen 9, SE-41296 Gothenburg, Swe- \\ den, yem@physics.gu.se, ${ }^{2}$ Department of Electronics, Institute of Information Technology \\ and Media, Mid Sweden University, Akademigatan 1, SE-831 25 Östersund, Sweden, An- \\ drei.Koptioug@miun.se
}

\begin{abstract}
In many engineering and biomedical problems there is a need to identify parameters of the systems from experimental data. A typical example is the biochemicalkinetics systems describing oncogenic hyperplasia where the dynamical model is nonlinear and the number of the parameters to be identified can reach a few hundreds. Solving these large-scale identification problems by the local- or globalsearch methods can not be practical because of the complexity and prohibitive computing time. These difficulties can be overcome by application of the nonsearch techniques which are much less computation- demanding. The present work proposes key components of the corresponding mathematical formulation of the nonsearch paradigm. This new framework for the nonlinear large-scale parameter identification specifies and further develops the ideas of the well-known approach of A. Krasovskii. The issues are illustrated with a concise analytical example. The new results and a few directions for future research are summarized in a dedicated section.
\end{abstract}

keywords: nonlinear dynamic system, non-search parameter, identification, Krasovskii method, biochemical kinetics

\section{Introduction}

In many engineering problems there is a need to identify parameters of systems from experimental data. This is equally true for both simple and complex systems (the latter are common in biomedical technology). A typical example is the biochemical-kinetics systems describing oncogenic hyperplasia (e.g., [1] - [3]), the first and therefore inevitable stage in development of any solid tumor. Oncogenic hyperplasia is a complex process related to many different types of molecules interacting with hyperplastic cells. In particular, these

Please use the following format when citing this chapter:

Mamontov, E., and Koptioug, A., 2006, in IFIP International Federation for Information Processing, Volume 202, Systems, Control, Modeling and Optimization, eds. Ceragioli, F., Dontchev, A., Furuta, H., Marti, K., Pandolfi, L., (Boston: Springer), pp. 269-278. 
interactions include the autocrine mechanism where the transforming-growthfactor- $\alpha$ molecules bind to the epidermal-growth-factor receptors at the cell surfaces (e.g.,[4]).

Oncogenic hyperplasia is also implicated in many other proliferative diseases (e.g., vascular, gastrointestinal, endocrine). The existing detailed models for the above phenomenon are ordinary differential equation (ODE) systems with a hundred or more equations (e.g., see the 104 equations in [4], Tables 1-3). These systems usually include hundreds of parameters to be identified. In other words, if $p$ is the vector of the parameters in the ODE system, then in the case of oncogenic hyperplasia

dimension $m$ of vector $p$ is on the order of a few hundreds.

This feature is also common in many in biomedical and engineering systems. Under condition (1), the parameter identification (sometimes applied to biochemical kinetics [5], [6]) based on the local or global search can not be practical because of the complexity and prohibitive computing time. The problem can be resolved by application of the techniques much less demanding in terms of the required computations. One of them is the nonsearch parameter-identification strategy.

The purpose of the present work is to present the nonsearch paradigm (i.e. the basic aspects of the mathematical formulation) for dynamical systems that have property (1) and which are related to oncogenic hyperplasia. The latter inevitably leads to nonlinear systems (e.g., [4]). Subsequently, the above paradigm should correspondingly extend the nonsearch settings well known (e.g., [7]) for linear and simple systems.

\section{Model}

The present work considers the following nonlinear ODE system

$$
d x / d t=f(t, x, p)
$$

where $t \in \mathbf{R}=(-\infty, \infty)$ is the time, $x \in \mathbf{R}^{n}(n \geq 1)$ is the state variable of the system under consideration, $p \in \mathbf{R}^{m}$ ( $m \geq 1$ ) is the vector of the system parameters discussed in Section 1 , and $f$ is the $n$-vector function sufficiently smooth on $\mathbf{R}^{n+m+1}$. As a rule, ODE (2) at every fixed $p$ possesses the following two properties:

- it is asymptotically stable in the Lyapunov sense in the large;

- moreover, it has the unique generally nonstationary solution, say, $\varphi_{s}(t, p)$, usually called the steady state solution, which is uniformly bounded for all $t \in \mathbf{R}$. 
The above stability implies that, if $\varphi\left(t, p, t_{0}, x_{0}\right)$ is the solution of $\operatorname{ODE}(2)$ with initial condition

$$
\left.x\right|_{t=t_{0}}=x_{0}
$$

where $t_{0} \in \mathbf{R}$ and $x_{0} \in \mathbf{R}^{n}$, then

$$
\varphi_{s}(t, p)=\lim _{t_{0} \rightarrow-\infty} \varphi\left(t, p, t_{0}, x_{0}\right), p \in \mathbf{R}^{m}, x_{0} \in \mathbf{R}^{n} .
$$

A discussion and references on ODE (2) with the above features can be found in [8]. The generalization of $\varphi_{s}(\cdot, p)$ for the diffusion stochastic processes is a nonstationary invariant DSP (paper [9] summarizes the results on these invariant processes).

Remark 1. In many cases, solution $\varphi_{s}(\cdot, p)$ can be obtained by means of the limit relation (4). Alternatively, one can determine $\varphi_{s}(t, p)$ as the unique solution of certain finite (i.e. nondifferential) equation (see [8]). The simplest approximation for it is

$$
f(t, x, p)=0, t \in \mathbf{R},
$$

which is the quasi-stationary version of ODE (2).

The next section proposes the necessary condition for the parameter identification to be meaningful.

\section{Identification and uniqiuiness of parameters.}

Identification of the parameter vector $p$ in ODE (2) is commonly implemented in the following way. One obtains the measurement data $\bar{x}_{s}$ for the steady-state solution $\varphi_{s}(t, \cdot)$ of (2), and then determines the actual value $p_{s}$ of $p$ (i.e. the value corresponding to the measured time dependence) by solving equation

$$
\bar{x}_{s}=\varphi_{s}\left(t, p_{s}\right), t \in \mathbf{R}
$$

for $p_{s}$.

Remark 2. The above extraction of $p_{s}$ from the steady-state solution makes $p_{s}$ independent of initial value $x_{0}$ in (3).

System (6) of $n$ equations is assumed to be uniquely solvable for $m$ entries of vector $p_{s}$, no matter what the values $n$ and $m$ are. Theory of nonlinear-system identification not always can deal with the question whether this is possible or not. To fill the gap, one can prove the following proposition.

Proposition 1. If function $\varphi_{s}$ is sufficiently smooth and equation (6) is solvable for $p_{s}$, then the equation has the only solution, if for any $p_{I}, p_{I I} \in \mathbf{R}^{m}$ 
such that $p_{I} \neq p_{I I}$, vectors $\int_{0}^{1} \frac{\partial \varphi_{s}\left(t, p_{I}+k\left(p_{I I}-p_{I}\right)\right)}{\partial p_{i}} \cdot d k, i=1, \ldots, m$, as functions of $t$, are linearly independent for all $t \in \mathbf{R}$ (scalars $p_{i}$ are the $i$-th entries of vector $p$ ).

The proof is based on the notion of a linear independence of vectors and representation

$\varphi_{s}\left(t, p_{I I}\right)-\varphi_{s}\left(t, p_{I}\right)=\left(p_{I I}-p_{I}\right) \int_{0}^{1}\left\{\partial \varphi_{s}\left[t, p_{I}+k\left(p_{I I}-p_{I}\right)\right] / \partial p\right\} d k$,

which results from the well-known formula (e.g. [10]). Criteria for the uniqueness in parameters similar to Proposition 1 are of a fundamental importance because it is impossible to consider the ODE-related identification in a meaningful way without a criterion of this kind.

\section{Nonsearch approach to the parameter identification}

In the nonlinear nonsearch approach (e.g. [11]), one obtains the measurement data $\bar{x}$ on the solution of initial-value problem (2), (3) where $p$ is equal to its actual value $p_{s}$, i.e.

$$
\begin{aligned}
& \bar{x}=\varphi\left(t, p_{s}, t_{0}, x_{0}\right), \\
& d \bar{x} / d t=f\left(t, \bar{x}, p_{s}\right) .
\end{aligned}
$$

To identify the parameter vector, i.e. to determine $p_{s}$, one implements the nonsearch procedure below.

Remark 3. The nonsearch procedure:

- allows $p$ in ODE (2) to depend on time $t$;

- describes the $t$-dependent $p$ with a dedicated ODE, say,

$$
d p / d t=g(t, x, p)
$$

with initial condition

$$
\left.p\right|_{t=t_{0}}=p_{0},
$$

where $g$ is the $m$-vector function sufficiently smooth in $\mathbf{R}^{n+m+1}$ and $p_{0} \in \mathbf{R}^{m}$;

- and ensures certain stability of ODE system (2), (9) which provides the behaviors

$$
\lim _{t \rightarrow \infty}(x-\bar{x})=0, \lim _{t \rightarrow \infty} p=p_{s}
$$

of the solution $(x, p)$ of a problem $(2),(9),(3), 10)$ for any initial vector $\left(x_{0}, p_{0}\right)$. 
The above recipe yields $p_{s}$ by the limit relation (11) thereby avoiding using any search technique.

This in principle resolves the problem associated with the high-number-ofparameters feature (1) typical in oncogenic hyperplasia (see Section 1).

In the above recipe, the stability is the key property to be assured. This can be done with the help of the theorem below.

Theorem 1. Let the following assumptions hold.

(A) Hypothesis of Proposition 1 is valid.

(B) ODE (2) is asymptotically stable in the Lyapunov sense in the large at any $t$-dependent sufficiently smooth $p$, and det $[\partial f(t, x, p) / \partial x] \neq 0$.

(C) State $x$ relaxes faster than parameter $p$ and there exists the integral manifold, say, $M$ for the Islow" variable $p$.

(D) ODE (9) is asymptotically stable in the Lyapunov sense in manifold $M$, and vector $\left.\lim \right|_{t-t_{0} \rightarrow \infty} p$ exists and is finite.

Then the nonsearch-identification recipe in Remark 3 is applicable.

The proof is not complicated. We only mention that the second equality in (11) follows from assumptions (A) and (D), and obvious relation $\lim _{t \rightarrow \infty} \bar{x}=$ $\bar{x}_{s}$, which stems from assumption (B) and equality (8).

Remark 4. In assumption (C) of Theorem 1, the manifold corresponds to the function $\varphi_{s}(t, p)$ in Section 1. It can be obtained as the solution of the finite equation resulting from the two-groups-of-components version of the method in reference [8], p.459 (cf., Remark 1). The simplest approximation for this equation is (5).

In the literature, there are no practical or even constructive criteria to verify or provide asymptotic stability in the large of general nonlinear ODEs. If function $g$ in (9) is nonlinear, it is especially difficult to design this function such that assumption (D) of Theorem 1 is assured. This is possible only in some particular cases.

In the general case, certain features critical to assumption (D) can be provided by the ideas of A. Krasovskii [11]. This topic is discussed in the next section.

\section{Specification of the Krasovskii method and new issues related to it}

The main relation in the method of Krasovskii is $\{[11]$, eqn. (3), (5), (6) $\}$

$$
d p / d t=g(t, x, p)=-d H / d p
$$


where $H\{[11]$, pp. 1852,1856$\}$ is the strictly convex function of $x-\bar{x}$ and $d H / d p$ (cf., [11], eqn. (11)) is the derivative along trajectories in the Islow"variable integral manifold $M$. Note, however, that the manifold idea in the Krasovskii paradigm is recognized but not formally developed (for instance, the corresponding derivation in reference [11], eqn. (12), (13), is not valid when $p$ depends on $t$ ). Following the above settings, for $H$ in (12) we consider the expression

$H(t, x, p)=\frac{1}{2}\left[(x-\bar{x})^{T} B\left(t, \bar{x}, p_{s}\right)(x-\bar{x})+\left(p-p_{s}\right)^{T} A\left(t, \bar{x}, p_{s}\right)\left(p-p_{s}\right)\right]$

where both matrices $A\left(t, \bar{x}, p_{s}\right)$ and $B\left(t, \bar{x}, p_{s}\right)$ are symmetric and positive definite. In contrast to (13), Krasovskii's expression for $H$ does not include either the second term in the brackets or the value $p_{s}$, which is unknown (cf., assumption (D) of Theorem 1). Vector $p_{s}$ in (13) is replaced below with a self-consistent predictor which is in a certain sense more accurate than $p$.

The derivative in (12) along trajectories in the Islow"-variable integral manifold $M$ is evaluated as

$$
d H(t, x, p) / d p=(\partial x / \partial p)^{T} B\left(t, \bar{x}, p_{s}\right)(x-\bar{x})+A\left(t, \bar{x}, p_{s}\right)\left(p-p_{s}\right)
$$

where the term $\partial x / \partial p$ on the right-hand side is estimated according to Remark 4 and equation (5). This results (see (12)) in

$$
d p / d t=-\left\{[C(t, x, p)]^{T} B\left(t, \bar{x}, p_{s}\right)(x-\bar{x})+A\left(t, \bar{x}, p_{s}\right)\left(p-p_{s}\right)\right\},
$$

where

$$
C(t, x, p)=-[\partial f(t, x, p) / \partial x]^{-1} \partial f(t, x, p) / \partial p .
$$

Equation (14) (see also (15)) generalizes the well-known Krasovskii equation $\{[11]$, eqn. (16)\}.

Reformulating (14) to answer the question if assumption (D) of Theorem 1 holds, one applies:

- the first equality in (11), which stems from assumption (B) of Theorem 1 and

- the approximate expression $x-\bar{x}=C\left(t, \bar{x}, p_{s}\right)\left(p-p_{s}\right)$, which stems from Remark 4, equation (5), and the first equality in (11).

The resulting form of (14) is

$$
d p / d t=-\left\{A\left(t, \bar{x}, p_{s}\right)+\left[C\left(t, \bar{x}, p_{s}\right)\right]^{T} B\left(t, \bar{x}, p_{s}\right) C\left(t, \bar{x}, p_{s}\right)\right\}\left(p-p_{s}\right)
$$

This is the specific version of the parameter ODE (9). 
Remark 5. The last term on the right-hand side of (14) is missing in the Krasovskii equation $\{[11]$, eqn. (16) $\}$. If it is not included in equation (16), this equation cannot be asymptotically stable when $m>n$ (since, in this case, ma$\operatorname{trix}\left[C\left(t, \bar{x}, p_{s}\right)\right]^{T} B\left(t, \bar{x}, p_{s}\right) C\left(t, \bar{x}, p_{s}\right)$ is singular, even if matrix $C\left(t, \bar{x}, p_{s}\right)$ is of the full rank, i.e. its rank is $n$ ).

Equation (16) is a linear ODE with time-dependent coefficients. Subsequently, its stability can be analyzed with the corresponding methods of the stability theory. Matrices $A\left(t, \bar{x}, p_{s}\right)$ and $B\left(t, \bar{x}, p_{s}\right)$ are to be determined to assure, firstly, the stability and secondly, the fact that vector $p$ in ODE (16) relaxes slower than vector $x$ in ODE (2) (which is required in assumption (C) of Theorem 1). We retain these topics for future research.

\section{Time average of parameters as a self-consistent predictor of the actual values}

Actual value $p_{s}$ is obtained (see (11)) as the time limit of $p$ from the solution of initial-value problem (2), (14), (3), (10). However, in the process of solving, $p_{s}$ is unknown and hence can not be employed in ODE (14). For this reason, $p_{s}$ in (14) is replaced with

$$
q=\frac{1}{t-t_{0}} \int_{t_{0}}^{t} p \cdot d s
$$

that results in

$$
d p / d t=-\left\{A(t, \bar{x}, q)+[C(t, \bar{x}, q)]^{T} B(t, \bar{x}, q) C(t, \bar{x}, q)\right\}(p-q) .
$$

Vector $q$ is a self-consistent predictor for $p_{s}$. Indeed, in contrast to the wellknown additional assumptions inherent in the parameter-shift technique (e.g. [7], Remark 8.4.1(ii) on p. 564), representation (17) involves only the time dependence of $p$ without extra assumptions. Predictor $q$ is also more preferable than any instantaneous value of $p$ because of the following.

Within the treatment based on the Theorem 1 , vector $\bar{x}$ in (18) is the measured value of the Ifast"-variable $x$ in ODE system (2), (18) whereas vector $p$ in (18) is the Islow"variable in the system (c.f., assumption (C) of the Theorem 1). In view of the coupling of (18) with the Ifast"-variable $x$, the Islow"variable $p$ is generally prone to rapid but comparatively short-living changes. Subsequently, the advantage of the ltime-average"form of (17) of predictor $q$ for $p_{s}$ is that it lfilters out"fast variations in the time dependence of instantaneous value $p$.

Also note, that $q$ defined by (17) is the solution of ODE

$$
d q / d t=(p-q) /\left(t-t_{0}\right)
$$


with initial condition

$$
\left.q\right|_{t=t_{0}}=p_{0}
$$

This description is equivalent to (17) and, thus, can be used instead of it.

Initial-value problem (18), (19), (10), (20) is the model developed in the present work to determine actual value $p_{s}=\lim _{t \rightarrow \infty} p$ and to deliver it to the initial- value problem (2), (3). This model is intended to serve as a computationally efficient alternative to the search-based parameter identification. It is probably the only practice-relevant option in the high-number-of-parameters case (1). Also note that ODE (18) is the more specific form of ODE (9) of Theorem 1 derived within a generalization of the ideas of [11].

\section{Example}

The specific model below exemplifies the analyses in this section and Section 5. Let the identical molecules of concentration $x$ and identical cells of concentration $u$ be suspended in a dispersion medium (e.g., the blood plasma). Assume that the cells release certain molecules, the time-dependence $u(t)$ of $u$ is known, and the molecules are disintegrated in the medium. Then these processes can be described with ODE (2), where $n=1\left(x_{1} \equiv x\right), m=2$, and $f(t, x, p)=-p_{1} x+p_{2} u(t)$, where $p_{1}$ and $p_{2}$ are the parameters. The problem is a determination of these parameters (generally dependent on the properties of the dispersion medium) from the measured data $\bar{x}=\bar{x}(t)$ (cf., (8)) on $x$.

According to the proposed treatment, the general model is system (2), (18), (19). In the example under consideration, the specific form of (2) is mentioned above. The form of (19) is fixed, independent of specific phenomena. The only issue is the specific form of (18). One can show (with the help of (15)) that this form is

$$
\left(\begin{array}{l}
p_{1}{ }^{\prime} \\
p_{2}{ }^{\prime}
\end{array}\right)=\left[\left(\begin{array}{cc}
A_{1} & 0 \\
0 & A_{2}
\end{array}\right)+\frac{B}{p_{1}^{2}}\left(\begin{array}{cc}
(\bar{x}(t))^{2} & -u(t) \bar{x}(t) \\
-u(t) \bar{x}(t) & (u(t))^{2}
\end{array}\right)\right]\left(\begin{array}{l}
p_{1}-q_{1} \\
p_{2}-q_{2}
\end{array}\right)
$$

where scalars $A_{1}>0$ and $A_{2}>0$ are the diagonal entries of symmetric and positive definite matrix $A\left(t, \bar{x}, p_{s}\right)$, which is assumed constant, and scalar $B>0$ is the $1 \times 1$ symmetric and positive definite matrix $B\left(t, \bar{x}, p_{s}\right)$. The mentioned scalars can be chosen as discussed in the text below Remark 5 , in particular, involving the well-known results of stability theory (e.g. [12]]. However, to begin with, one can estimate them in line with the issues in ([7], Chapter 4).

Also note that the manifold in assertion (C) of Theorem 1 exists and is described as $x=\int_{-\infty}^{t}\left\{\exp \left[-\int_{s}^{t}\left(p_{1}(\nu)\right)^{-1} d \nu\right] p_{2}(s) u(s)\right\} d s$. The condition when this manifold is the one for "slow" vector $\left(p_{1}, p_{2}\right)$ can be obtained by means of results of [8]. 
The above example illustrates the proposed innovative method, based upon the ideas of Theorem 1. The new approach presents nontrivial extension of the well+known techniques available in the literature (e.g., [7], Chapter 4).

\section{Concluding remarks and directions for further research}

The main new results developed in the present work include:

- Proposition 1 (on the uniqueness in the parameters);

- Theorem 1 (on the qualitative features of the model for the nonsearch identification of parameters of nonlinear systems);

- three new issues on a specification of the Krasovskii method:

- the multi-aspect role of the Islow"-variable manifold,

- the dissipative term in the ODE for the parameter vector,

- the self-consistent predictor for the actual values of parameters;

- a generic model for the nonsearch identification of nonlinear-system parameters (initial-value problem (2), (18), (19), (3), (10), (20)).

A few directions for future research are: (1) application of the present results to numerical parameter identification in biochemical kinetics of oncogenic hyperplasia in can-cer and other proliferative diseases, (2) generalization to Itô's stochastic nonlinear differential equations taking into account the influence of random noises (this can make use of the analytical-numerical methods developed for high-dimensional nonlinear stochastic systems [13]), and (3) extension of the stability-based nonsearch optimization/identifica-tion to new areas such as self-navigated truly autonomous (fully GPS-independent) robots capable of delivering light load, or intercepting mobile targets (e.g., [14]). Future rigorous analysis of the mathematical formulation proposed in this work will contribute to specification of the details which can facilitate practical application of the present approach.

\section{References}

[1] A. V. Koptioug, E. Mamontov. Toward prevention of hyperplasia in oncogeny and other proliferative diseases: The role of the cell genotoxicity in the model-based strategies. Abstracts: 7th Ann. Conf.: Functional Genomics -From Birth to Death. Göteborg, Sweden, August 19-20, 2004.

[2] A. V. Koptioug, E. Mamontov, Z. Taib, M. Willander. The phase-transition morphogenic model for oncogeny as a genotoxic homeostatic dysfunction: Interdependence of modeling, advanced measurements, and numerical simulation. Abstracts: ICSB2004, 5th Int. Conf. Systems Biology. Heidelberg, Germany, October 9-13, 2004. 
[3] K. Psiuk-Maksymowicz, E. Mamontov. The time-slice method for rapid solving the Cauchy problem for nonlinear reaction-diffusion equations in the competition of homeorhesis with genotoxically activated oncogenic hyperplasia. Abstracts: The European Conference on Mathematical and Theoretical Biology. Dresden, Germany, July 18-22, 2005.

[4] J. H. Miller, F. Zheng. Large-scale simulations of cellular signaling processes. Parallel Computing 30: 1137-1149, 2004.

[5] J. Yen, J. C. Liao, B. Lee, D. Randolph. A hybrid approach to modeling metabolic systems using a genetic algorithm and simplex method. IEEE Trans. Systems, Man, and Cybernetics 28(2): 173-191, 1998.

[6] C.-Y. F. Huang, J. E. Ferrel, Jr. Ultrasensitivity in the mitogen-activated protein kinase cascade. Proc. Natl. Acad. Sci. USA 93(September): 10078-10083, 1996.

[7] P. A. Ioannou, J. Sun. Robust Adaptive Control. Prentice- Hall, Upper Saddle River, NJ, USA, 1996.

[8] Y. V. Mamontov, M. Willander. Asymptotic method of finite equation for bounded solutions of nonlinear smooth ODEs. Mathematica Japonica 46(3): 451-461, 1997.

[9] E. Mamontov, Nonstationary invariant distributions and the hydrodynamic- style generalization of the Kolmogorov-forward/Fokker-Planck equation. Appl. Math. Lett. 18(9): 976-982, 2005.

[10] J. M. Ortega, W. C. Rheinboldt. Iterative Solution of Nonlinear Equations in Several Variables. Academic Press, New York, 1970.

[11] A. A. Krasovskii. Optimal algorithms in identification problem with an adaptive model. Automation and Remote Control (May 10): 1851-1857, 1977.

[12] N. Rouche, P. Habets, and M. Laloy. Stability Theory by Liapunov's Direct Method. Springer-Verlag, 1977.

[13] Y. V. Mamontov, M. Willander. High-Dimensional Nonlinear Diffusion Stochastic Processes. Modeling for Engineering Applications. World Scientific, Singapore, 2001.

[14] E. Mamontov, A. Koptioug, M. Mångård, K. Marti. Asymptotic trajectory matching in self-navigation of autonomous manless interceptors: Nonsearch method and a formulation of the functional optimization of the stability of random systems. Abstracts: 5 th MATHMOD Vienna Conf., Special Session "Stochastic Optimization Methods", Chair: K. Marti. Austria, Vienna, Vienna Univ. of Technol., 7-9 February, 2006. http://www.mathmod.at/. 